\title{
12. On the Spatial and Temporal Summation of the Postsynaptic Potential of the Motoneuron of the Spinal Cord of Cats
}

\author{
Jiro Tatsuno and Yutaka Matsumoto \\ Department of Neuropsychiatry, School of Medicine, \\ Chiba University
}

It is probable that the stimulation of the motor cortex might influence the postsynaptic potential of the motor cell of the spinal cord initiated by the afferent impulses from the annulospiral endings of the muscle receptors.

In this experiment, adult cats, very lightly anesthetized by pentobarbital, are used and the contralateral posterior sigmoid gyrus is exposed and the ventral roots of L4-S1 are cut after laminectomy. Recordings are made at the central cut ends of the functionally isolated single filaments of the ventral roots which show the monosynaptic reflex to the stimulation of the peripheral nerve to $\mathrm{m}$. gastrocunemius.

The intensity of both stimulations, on the surface of the motor cortex and at the muscle nerve to $\mathrm{m}$. gastroc., is adjusted just under the threshold, so that the monosynaptic reflex does not occur by each one of them. But when the peripheral stimulation is preceded by the central stimulation in appropriate time interval, monosynaptic reflex can be observed. As the interval increases, the response time after the secondary stimulation decreases and then increases. (Fig. 1)

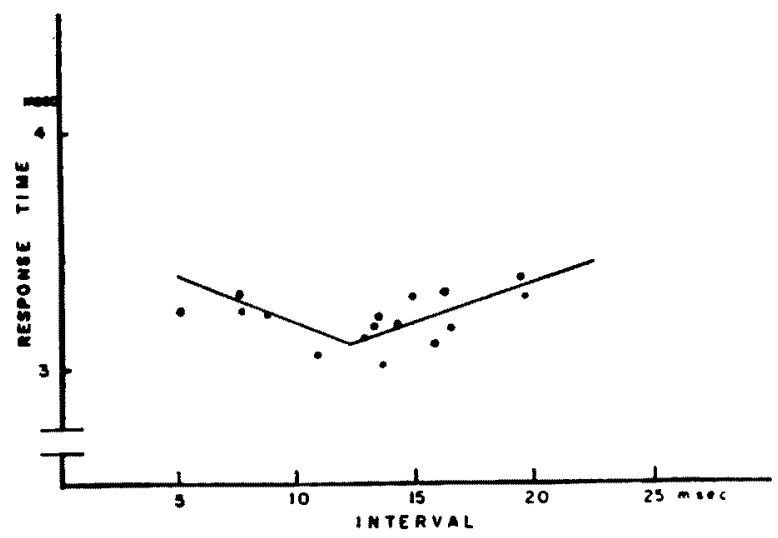

Fig 1

The time course of the effect on the motoneuron of the spinal cord by the stimulation of the motor cortex is deducible according to the proposition that the postsynaptic potentials produced by each stimulations summate temporally and spatially on the membrane of the motor cell of spinal cord. 
If the types of stimulations change, the modes of summation of the postsynaptic potentials are variable, and it needs further research to make clear the relationship of them.

\title{
13. Restoration of Diaphragmatic Contraction Following Intercosto-Phrenic Nerve Anastomosis
}

\author{
Tokuo Ogawa, Eiichi Terada, Mamoru Kobayashi and Kentaro Takagi \\ Department of Physiology, School of Medicine, Nagoya \\ University, Nagoya,
}

In dogs, the distal stump of a cut phrenic nerve was anastomosed with the central cut end of the 6th intercostal nerve of the same side. Approximately five months after the operation, spontaneous inspiratory contraction of the diaphragm recurred. The intact 6 th intercostal nerve conducts both inspiratory and expiratory impulses, and yet restored diaphragmatic contraction was concomitant with inspiratory movement of the thorax.

In an attempt to clarify the process of this outwardly purposive regeneration, dogs were examined 114 to 221 days after the anastomosis by means of electromyography and visual observations of the diaphragm. At a very early stage of regeneration, both inspiratory and expiratory action potentials were recorded from the diaphragm, although the former exceeded the latter in amplitudes and number of motor units. No spontaneous contraction of the diaphragm was seen at this stage. In the course of time, inspiratory action potentials became further dominant as compared with expiratory ones, and inspiratory contraction of the diaphragm became visible, also. In approx, 150 days after the anastomosis, expiratory action potentials were no longer recorded from the diaphragm, but only inspiratory ones remained. Even at this stage, both inspiratory and expiratory action potentials were recorded from the 6 th intercostal muscle proximal to the site of the nerve anastomosis.

From the above observations, the following can be concluded: Intercostophrenic nerve anastomosis is followed by regeneration of fibers of the intercostal nerve which transmit inspiratory impulses as well as fibers which act expiratorily, but the former always exceed the latter which eventually lose their function. The former may be assumed to regenerate more readily than the latter, since the phrenic nerve originally contains fibers which act inspiratorily. Furthermore, afferent impulses from alveoli and/or the diaphragm may participate in the process of "fadeout" of expiratory impulses to the diaphragm. The mechanisms remain to be investigated. 